\title{
DIFFERENCE SCHEMES FOR GENERALIZED SOLUTIONS OF SOME ELLIPTIC DIFFERENTIAL EQUATIONS, II
}

\author{
HOANG DINH DUNG
}

\begin{abstract}
The approximate methods for the problems of differential equations with non-regular data are studied by some authors. For example, in $[1-3,6,7]$ are considered the cases of data belonging to the Sobolev spaces $W_{p}^{l}(G)$. In this paper, which is a continuation of [4], we consider the difference schemes for solutions of some elliptic problems in the case where the region of definition for variable has arbitrary form. In the last section the result is generalized to a class of problems with data defined by the continuous linear functionals in $W_{2}^{(-l)}(G)$.
\end{abstract}

\section{DIFFERENCE SCHEME FOR THE DIRICHLET PROBLEM OF POISSON EQUATION}

Consider the following Dirichlet problem:

$$
\begin{aligned}
\Delta u & =-f(x), \quad x \in G, \\
u(x) & =0, \quad x \in \partial G .
\end{aligned}
$$

To simplify the exposition, assume that $G$ is a convex region in $R^{2}$ with $\partial G \in C^{2}$. We shall keep some notations in [4], [7].

Let $R_{h}$ be a rectangle grid covered the $x$-plane and defined by

$$
R_{h} \equiv\left\{x=\left(x_{1}, x_{2}\right): x_{i}=x^{\left(j_{i}\right)}=j_{i} h_{i}, j_{i}=0, \pm 1, \pm 2, \ldots, i=1,2\right\}
$$

where the straight lines $x_{i}$ are the parallels to the coordinate lines, $h_{i}$ are positive mesh sizes in the $x_{i}$-directions, $i=1,2$, respectively. Denote by $\omega=R_{h} \cap G$ the set of all gridpoints in $G$, and by $\gamma=R_{h} \cap \partial G$ the set of boundary gridpoints, by $\gamma_{i}^{+}$and $\gamma_{j}^{-}$the set of right and left boundary gridpoints in the $x_{i}$-directions respectively. Let $\omega_{\gamma}$ be the subset of interior netpoints that the lie in the neighbourhood of $\partial G, \omega_{0} \equiv \omega \backslash \omega_{\gamma}, \bar{\omega} \equiv \omega \cup \gamma$.

Let us introduce a supplementary grid of the parallels $x_{\beta}$ to the lines $x_{i}$ :

$$
x_{\beta} \equiv x_{i}^{\left(j_{i}+0.5\right)}=0.5\left(x_{i}^{\left(j_{i}\right)}+x_{i}^{\left(j_{i}+1\right)}\right) .
$$

Let every gridpoint $x \in \omega$ be corresponding to the subregion $\widetilde{e}(x) \in G$ bounded by the straight lines $x_{\beta}=x_{i}^{\left(j_{i}+0.5\right)}, i=1,2$. If $x \in \omega_{\gamma}, \tilde{e}(x)$ is limited by not only the $x_{\beta}$ but also an arc of the curve $\partial G$. The boundary segments $x_{\beta}$ of $\tilde{e}(x)$ perpendicular to the coordinate lines $O x_{i}$ are denoted by $l_{i}^{( \pm 0.5)}$, $i=1,2$, respectively.

Denote by $x^{\left( \pm 1_{i}\right)}, i=1,2$, the neighbourhood netpoints of the netpoint $x \in \bar{\omega}$ in the $x_{i}$-direction, $h_{i}^{\left( \pm 1_{i}\right)} \equiv\left|x_{i}^{\left( \pm 1_{i}\right)}-x_{i}\right|, x_{i}$ and $x_{i}^{\left( \pm 1_{i}\right)}$ being the coordinates of the netpoints $x$ and $x^{\left( \pm 1_{i}\right)} \in \bar{\omega}$ respectively. We see that there are the differences of steplengths $h^{( \pm 0.5)}$ and $h_{i}$ only in the neighbourhoods of $\partial G$.

The points of intersection of the straight lines $x_{i}=x_{i}^{\left(j_{i}\right)}$ with $x_{\beta}=x_{i}^{\left(j_{i} \pm 0.5\right)}$ are denoted by $x^{\left( \pm 0.5_{i}\right)}$ that are called the stream gridpoints in the $x_{i}$-direction. Denote by $\omega_{i}^{\prime}$ the set of these points, $\omega^{\prime} \equiv \omega_{1}^{\prime} \cup \omega_{2}^{\prime}$. 
Let every gridpoint $x^{\left( \pm 0.5_{i}\right)}$ correspond to a following area, $i=1,2$,

$$
e_{i}\left(x^{\left( \pm 0.5_{i}\right)}\right)=\left\{\zeta=\left(\varsigma_{1}, \varsigma_{2}\right): x_{i}<\varsigma_{i}<x_{i}+h_{i},\left|x_{\beta}-\varsigma_{\beta}\right|<0.5 h_{\beta}, \quad \beta=3-i\right\} .
$$

Let $x \in \omega_{\gamma}$ and in the area $\tilde{e}(x)$ the segment $\bar{\Delta} l$ correspond to the arc $\Delta \Gamma$ of $\partial G(\Gamma \equiv \partial G)$. Denote by $e(x)$ the area bounded by the segments $l_{i}^{( \pm 0.5)}$ and $\bar{\Delta} l$. Note that, by assumptions for $G$, with $x \in \omega_{\gamma}$ the different value between the areas $\tilde{e}(x)$ and $e(x)$ is equal to $O(h)$, where $|h|^{2}=h_{1}^{2}+h_{2}^{2}$.

\subsection{Construction of difference schemes}

The generalized solution of the problem (1) is considered in the spaces $W_{2}^{m}(G), m=2,3$. As in [4] the generalized solution (denoted by the $G S) u(x)$ satisfies the following equalities:

$$
P u \equiv \iint_{G} \Delta u(x) v(x) d x=-\iint_{G} f(x) v(x) d x, \forall v(x) \in L_{2}(G) ; u(x)=0, x \in \partial G .
$$

Let $x \in \omega_{\gamma}$. For deriving finite-difference methods, we may take the solution of (2) in the neighbourhood area $e(x)$ of the gridpoint $x$ by the form:

$$
\begin{aligned}
P^{e} u & =\frac{1}{h_{1} h_{2}} \iint_{e(x)} \alpha\left(\zeta_{1}, \zeta_{2}\right) \Delta u(\zeta) d \zeta=\frac{1}{h_{1} h_{2}} \iint_{e(x)}\left[\sum_{i=1}^{2} \frac{\partial u}{\partial \zeta_{i}}\left(\alpha \frac{\partial u}{\partial \zeta_{i}}\right)-\sum_{i=1}^{2} \frac{\partial \alpha}{\partial \zeta_{i}} \frac{\partial u}{\partial \zeta_{i}}\right] d \zeta \\
& =-\frac{1}{h_{1} h_{2}} \iint_{e(x)} \alpha(\zeta) f(\zeta) d \zeta \equiv-R f,
\end{aligned}
$$

where $v(x)=\left(h_{1} h_{2}\right)^{-1} \alpha(x), \alpha(x)= \begin{cases}\frac{1}{4 \pi h_{1}^{m-1} h_{2}^{m-1}} \exp \left\{-\frac{x_{1}^{2}+x_{2}^{2}}{4 h_{1}^{m} h_{2}^{m}}\right\}, & x \in e, \\ 0, & x \in \bar{G} \backslash e .\end{cases}$

From (3), applying the Green-Ostrogradski formula one has

$$
\begin{aligned}
P^{e} u= & \frac{1}{h_{1} h_{2}}\left\{\sum_{i=1}^{2} l_{i}^{(0.5)} \bar{\omega}_{i}^{\left(+0.5_{i}\right)}-\sum_{i=1}^{2} l_{i}^{(0.5)} \bar{\omega}_{i}^{\left(-0.5_{i}\right)}+\Delta l \beta(x) \bar{\omega}^{(0)}\right\} \\
& -\frac{1}{h_{1} h_{2}} \iint_{e(x)} \sum_{i=1}^{2} \frac{\partial \alpha}{\partial \zeta_{i}} \frac{\partial u}{\partial \zeta_{i}} d \zeta=-R f, x \in \omega_{\gamma} ; u(x)=0, x \in \gamma,
\end{aligned}
$$

where

$$
\bar{\omega}_{i}^{\left( \pm 0.5_{i}\right)}=\frac{e(x)}{l_{i}^{( \pm 0.5)}} \int_{l_{i}^{( \pm 0.5)}} \alpha \frac{\partial u}{\partial x_{i}} d l, \quad \bar{\omega}^{(0)}=\frac{1}{\Delta l} \int_{\frac{\Delta l}{\partial n}} \alpha \frac{\partial u}{\partial n} d l,
$$

the net function $\beta(x)$ is equal to 1 as $x \in \omega_{\gamma}$ and is zero as $x \in \omega_{0}$, the lengths of segments $l_{i}^{( \pm 0.5)}$ are denoted also by $l_{i}^{( \pm 0.5)}, \bar{\omega}^{(0)}$ is calculated by a contour integral of the first kind. The notation " $\sum_{t}^{\prime \prime}$ "signifies that this sum has no the $i$-th summand corresponding to the $l_{i}^{( \pm 0.5)}=\emptyset$ respectively, $\vec{n}$ being the outer normal to $\partial e$.

Note that if the netpoint $x \in \omega_{0}$, one has the form of $P^{e} u$ analogous to (4) in which the sum $\sum$ has no the sign "' " and $l_{3-i}^{( \pm 0.5)}, i=1,2$, are replaced by $h_{i}$ respectively.

Now, to construct the difference schemes one may do in the same way as in Section 2.1 for the net problem (8), [4]. Thus, by (4) we obtain the following difference approximations analogous to (9) and (12) in [4] respectively:

$$
\begin{aligned}
& K \bar{y} \equiv-\left(a_{1} \bar{y}_{x_{1}}\right)_{\widehat{x}_{1}}-\left(a_{2} \bar{y}_{x_{2}}\right)_{\widehat{x}_{2}}+\frac{1}{h_{1} h_{2}} \iint_{e(x)} \sum_{i=1}^{2} \alpha_{\bar{x}_{i}}(x) \bar{y}_{\bar{x}_{i}}(x) \bar{y}_{\bar{x}_{i}}(x) d \zeta=\varphi(x) \equiv R f(x), \\
& x \in \omega ; \quad \bar{y}(x)=0, \quad x \in \gamma,
\end{aligned}
$$

and

$$
L \widehat{y}=-\widehat{y}_{x_{1} \bar{x}_{1}}-\widehat{y}_{x_{2} \bar{x}_{2}}=\varphi(x), x \in \omega ; \hat{y}(x)=0, x \in \gamma,
$$


where

$$
\begin{aligned}
& y_{x_{i}}^{\left(+0.5_{i}\right)}=\frac{y^{\left.+1_{i}\right)}-y}{h_{i}^{(+0.5)}}, y_{x_{i}}^{\left.-0.5_{i}\right)}=\frac{y-y^{\left(-1_{i}\right)}}{h_{i}^{(-0.5)}}, \quad y_{\widehat{x}_{i}}=\frac{y^{\left(+0.5_{i}\right)}-y^{\left(-0.5_{i}\right)}}{h_{i}}, \\
& a_{i}^{\left( \pm 0.5_{i}\right)}=\frac{1}{l_{i}^{\left( \pm 0.5_{i}\right)}} \int_{l_{i}^{\left( \pm 0.5_{i}\right)}} \alpha(\zeta) d l \text { for } l_{i}^{\left( \pm 0.5_{i}\right)} \neq 0, a_{i}^{\left( \pm 0.5_{i}\right)}=\frac{1}{\Delta l} \int_{\frac{1}{\Delta l}} \alpha(\zeta) d l \text { for } l_{i}^{\left( \pm 0.5_{i}\right)}=0 .
\end{aligned}
$$

Note that the integrals should be taken along the segments $l_{i}^{( \pm 0.5)}$ and $\overline{\Delta l}$ lying inside the region $\bar{G}$. For $x \in \omega_{0}$ one has the formula similar to (6).

\subsection{Estimation of the convergence rate}

We shall estimate the method error and the approximate error of the scheme (7) and (6).

1.2.1. Consider first the difference scheme (7). The left-hand side of the difference equation (7) coincides with a standard fivepoints approximation for the one of the differential equation (1) in the case of the variable region $G$ of any form. Consider now the convergence of the approximate solution $\widehat{y}$ to the $G S u$ of form (4). Denote the method error by $z \equiv \widehat{y}-u$. By (7) one has

$$
L z=\Psi(x), x \in \omega ; z(x)=0, x \in \gamma,
$$

where $\Psi(x)$ is the approximate error of the scheme (7): $\Psi(x)=\varphi(x)-L U$. Then, using the expression (4) of $\varphi=R f$ we get

$$
L z=\Psi=\sum_{i=1}^{2} \eta_{i_{\hat{x}_{i}}}+\eta_{0}
$$

where

$$
\begin{aligned}
\eta_{i}^{\left( \pm 0.5_{i}\right)}=u_{x_{i}}^{\left( \pm 0.5_{i}\right)}-\bar{w}_{i}^{\left( \pm 0.5_{i}\right)} \text { if } l_{i}^{\left( \pm 0.5_{i}\right)}=h_{3-1} \\
\eta_{i}^{\left( \pm 0.5_{i}\right)}=u_{x_{i}}^{\left( \pm 0.5_{i}\right)}-\bar{w}_{i}^{\left( \pm 0.5_{i}\right)}+\left(1-\frac{l_{i}^{\left( \pm 0.5_{i}\right)}}{h_{3-i}}\right)\left(\bar{w}_{i}^{\left( \pm 0.5_{i}\right)}-\tilde{w}_{i}\right) \text { if } 0<l_{i}^{\left( \pm 0.5_{i}\right)}<h_{3-i} \\
\eta_{i}^{\left( \pm 0.5_{i}\right)}=u_{x_{i}}^{\left( \pm 0.5_{i}\right)}-\tilde{w}_{i} \text { if } l_{i}^{\left( \pm 0.5_{i}\right)}=0 \\
\tilde{w}_{i}=\frac{1}{\Delta l} \int \frac{\partial u}{\Delta l} \alpha \frac{\partial u}{\partial x_{i}} d l, \quad \eta_{0}=\frac{1}{h_{1} h_{2}} \iint_{e(x)} \sum_{i=1}^{2} \frac{\partial \alpha}{\partial \zeta_{i}} \frac{\partial u}{\partial \zeta_{i}} d \zeta .
\end{aligned}
$$

Now, to obtain a priori estimation, let us scalar multiply both sides of (9) by $z(x)$ and, then, arguing by the same way as in $[4$, Sec. 2.2], we get

$$
\|z\|_{1, \omega} \leq M\left(\left\|\eta_{1}\right\|_{0, \omega^{\prime}}+\left\|\eta_{2}\right\|_{0, \omega^{\prime}}+\left\|\eta_{0}\right\|_{0, \omega^{\prime}}\right)
$$

where $M$ is a constant independent of $h$ and $z(x)$,

$$
\begin{aligned}
\|v\|_{1, \omega}^{2} & =\|v\|_{0, \omega}^{2}+\|\nabla v\|_{0, \omega^{\prime}}, \quad\|v\|_{0, \omega} \equiv \sqrt{(v, v)} \\
\|\nabla v\|_{0, \omega^{\prime}}^{2} & \equiv(\nabla v, \nabla v)^{\prime}, \quad \nabla v\left(x^{\left( \pm 0.5_{i}\right)}\right) \equiv v_{x_{i}}^{\left( \pm 0.5_{i}\right)} \text { for } x^{\left( \pm 0.5_{i}\right)} \in \omega_{i}^{\prime},
\end{aligned}
$$

$(u, v)$ is the scalar product on the set of net functions $\stackrel{\circ}{H}_{h}:(u, v) \equiv \sum_{x \in \omega} h_{1} h_{2} u(x) v(x),(u, v)^{\prime}$ is the scalar product on the set of functions defined on the net $\omega^{\prime}$ of stream gridpoints $H_{h}^{\prime}$ :

$$
(u, v)^{\prime} \equiv \sum_{i=1}^{2} \sum_{x^{\left( \pm 0.5_{i}\right)} \in \omega_{i}^{\prime}} h_{i}^{\left( \pm 0.5_{i}\right)} h_{3-i} u\left(x^{\left( \pm 0.5_{i}\right)}\right) v\left(x^{\left( \pm 0.5_{i}\right)}\right) .
$$

The estimation of summands in the right-hand side of (14) is analogous to that of (18) in Section 2.2 [4], and one has 


$$
\|z\|_{1, \omega} \leq M|h|^{m-1}\|u\|_{m, \mathfrak{\circ}}+M|h|\|\underline{\|}\|_{2, \widehat{G}}
$$

where $m=2,3 ; \stackrel{\circ}{G}=\bigcup_{x^{\prime} \in \omega_{0}^{\prime}} e_{i}\left(x^{\prime}\right), \widehat{G}=\bigcup_{x^{\prime} \in \omega_{\gamma}^{\prime}} \widehat{e}_{i}\left(x^{\prime}\right), x^{\prime}=x^{( \pm 0.5)}, i=1,2$, $\omega_{0}$ being the subset of gridpoints $x^{\prime}$ that $e_{i}\left(x^{\prime}\right) \subset G$ (Fig. a), $\omega_{\gamma}^{\prime} \equiv \omega^{\prime} \backslash \omega_{0}^{\prime}$; If $x^{\prime} \in \omega_{\gamma}^{\prime}$, then $e_{i}\left(x^{\prime}\right) \equiv \widehat{e}_{i} \cup \bar{e}_{i}$ with $\widehat{e}_{i} \subset G$ and $\bar{e}_{i} \not \subset G$ (Fig. b).

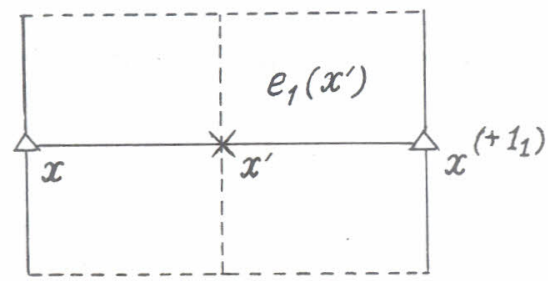

Fig. a

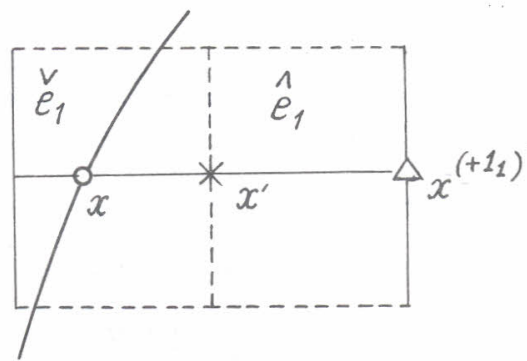

Fig. $b$

The set $\widehat{G}$ can be bounded by a boundary strip $G_{\varepsilon}$ with its width $\varepsilon=M|h|$. Then, if $u \in W_{2}^{3}(G)$ one has the following estimation [7]

$$
\|u\|_{2, \widehat{G}} \leq M|h|^{1 / 2}\|u\|_{3, G} .
$$

Finally, if follows from (15) and (16)

$$
\|\widehat{y}-u\|_{1, \omega} \leq M|h|^{m / 2}\|u\|_{m, G},
$$

where $m=2,3$; the constant $M$ is independent of $h$ and $u(x)$.

1.2.2. Consider now the difference scheme (6). By the same way as we did for the scheme (9) in the Section 2.2 [4], and for the scheme (7) above, with employing (17) one obtains the following result

Theorem 1. Let $\alpha(x) f(x) \in L_{2}(G)$. Then the solution $\bar{y}$ of the scheme (6) converges to the $G S$ (4) $u(x)$ of the problem (1) in the grid norm $W_{2}^{1}(\omega)$ with the rate $O\left(|h|^{m / 2}\right)$ that is, there is a number $M$ such that

$$
\|\bar{y}-u\|_{1, \omega} \leq M|h|^{m / 2}\|u\|_{m, G},
$$

where the constant $M$ is independent of $h$ and $u(x), m=2,3$.

\section{ELLIPTIC DIFFERENTIAL EQUATION OF THE SECOND ORDER WITH VARIABLE COEFFICIENTS}

Consider the elliptic problem

$$
P_{u} \equiv \sum_{i=1}^{2} \frac{\partial}{\partial x_{i}}\left(k_{i}(x) \frac{\partial u}{\partial x_{i}}\right)=-f(x), x \in G ; u(x)=0, x \in \partial G,
$$

where $G$ is defined as in the problem $(1), k_{i}(x) \in C(\bar{G}), i=1,2$,

here $C_{i}$ being constants.

$$
0<C_{1} \leq k_{i}(x) \leq C_{2}, \quad x \in \bar{G},
$$

\subsection{Construction of difference scheme}

Consider the $G S$ of the problem $(19) u(x)$ in the space $W_{2}^{m}(G) \cap \stackrel{\circ}{W}_{2}^{1}(G)$ satisfying the equality:

$$
\iint_{G} \sum_{i=1}^{2}\left(k_{1}(x) \frac{\partial u}{\partial x_{i}}\right) v(x)=-\iint_{G} f(x) v(x) d x, \quad \forall v(x) \in \stackrel{\circ}{W}_{2}^{m}(G)
$$


From the last equation, arguing as in Sections 1.1 and 3.1, [4], one has the following net problem for the $G S$ of the problem (19):

$$
\begin{aligned}
P^{e} u & =\frac{1}{h_{1} h_{2}}\left\{\sum_{i=1}^{2} l_{i}^{(+0.5)} \breve{\omega}_{i}^{\left(+0.5_{i}\right)}-\sum_{i=1}^{2} l_{i}^{\left(-0.5_{i}\right)}+\Delta l \beta(x) \breve{\omega}^{(0)}\right\} \\
& -\frac{1}{h_{1} h_{2}} \iint_{e(x)}^{2} \sum_{i=1}^{2} k_{i} \frac{\partial \alpha}{\partial \zeta_{i}} \frac{\partial u}{\partial \zeta_{i}} d \zeta=R f, \quad u(x)=0, \quad x \in \gamma,
\end{aligned}
$$

where $R f \equiv \varphi$ and $\alpha(x)$ have the form (3), $\beta(x)$ is defined as in (4),

$$
\bar{\omega}_{i}^{( \pm 0.5)}=\frac{1}{l_{i}^{( \pm 0.5)}} \int_{l_{i}^{( \pm 0.5)}} k_{i} \alpha \frac{\partial u}{\partial x_{i}} d l, \quad \bar{\omega}^{(0)}=\frac{1}{\Delta l} \int_{\frac{1}{\Delta l}} k_{i} \alpha \frac{\partial u}{\partial l} d l .
$$

By (22), in a manner analogous to the proof of the forms (6) and (7) one obtains the following difference schemes of the net problem (22):

$$
\begin{aligned}
& \tilde{K} y \equiv-\sum_{i=1}^{2}\left(b_{i} \bar{y}_{x_{i}}\right)_{\widehat{x}_{i}}+\frac{1}{h_{1} h_{2}} \iint_{e(x)} \sum_{i=1}^{2} k_{i}(x) \alpha_{\bar{x}_{i}} \bar{y}_{\bar{x}_{i}}(x) d \zeta=\varphi(x) \breve{y}(x)=0, x \in \gamma, \\
& \tilde{L} \hat{y} \equiv-\sum_{i=1}^{2}\left(d_{i} \widehat{y}_{x_{i}}\right)_{\widehat{x}_{i}}=\varphi(x) ; \hat{y}(x)=0, \quad x \in \gamma,
\end{aligned}
$$

where

$$
\begin{aligned}
& b_{i}^{( \pm 0.5)}=\frac{1}{l_{i}^{( \pm 0.5)}} \int l_{i}^{( \pm 0.5)} k_{i}(\zeta) \alpha(\zeta) d l \text { for } l_{i}^{( \pm 0.5)} \neq 0, \\
& b_{i}^{( \pm 0.5)}=\frac{1}{\Delta l} \frac{\int}{\Delta l} k_{i}(\zeta) \alpha(\zeta) d l \text { for } l_{i}^{( \pm 0.5)}=0 \\
& d_{i}^{( \pm 0.5)}=\frac{1}{l_{i}^{( \pm 0.5)}} \int_{l_{i}^{( \pm 0.5)}} k_{i}(\zeta) d l \text { for } l_{i}^{( \pm 0.5)} \neq 0, \\
& d_{i}^{( \pm 0.5)}=\frac{1}{\Delta l} \int_{\frac{\Delta l}{\Delta l}} k_{i}(\zeta) d l \text { for } l_{i}^{( \pm 0.5)}=0 .
\end{aligned}
$$

\subsection{Estimate of convergence rate}

By (22)-(24), arguing as in the proof of the Theorem 1, we have following.

Theorem 2. Let $k_{i}(x) \in W_{\infty}^{m-1}(G), i=1,2$, satisfying the condition $(20), m=2,3 ; \alpha(x) f(x) \in$ $L_{2}(G)$. Then the solution $y$ of the scheme (23) or (24) $(y=\breve{y}$ or $\hat{y})$ converges to the $G S(22) u$ of the problem (19) in the net norm $W_{2}^{1}(\omega)$ with the rate $O\left(|h|^{m / 2}\right)$, that is there is a number $M$ such that

$$
\|y-u\|_{1, \omega} \leq M|h|^{m / 2}\|u\|_{m, G},
$$

where the constant $M$ is independent of $h$ and $u(x)$.

\section{Remark}

a. For simplicity of presentation, the homogeneous boundary condition was considered. The Theorems 1 and 2 are also valid in the case where $u(x)=g(x), x \in \partial G$.

b. Some generalizations given in Section 2.3, [4] are also true for the problems (1) and (19):

- The Theorems 1 and 2 are also valid, if in the formulas (2) and (21) of the $G S u(x), v f(x)$ is any function in the space $D(G)$ of Schwartz basic functions [8]. 
- It is known (see [5], [9], etc.) that the right hand side of differential equations in the environment problems may be a functional (e.g., $f$ is the Dirac delta functions $\delta$ ). The estimates (17), (18) and (25) are obtained with the assumption $f \in L_{2}(G)$, now we show that the results may be generalized to the equations with right-hand side $f \in W_{2}^{(-l)}(G)$ - the space of continuous linear functionals on the space $\stackrel{\circ}{W}_{2}^{l}(G), l$ is a nonnegative integer. Indeed, by this assumption, $f(x) \in D^{\prime}(G)$ the space of Schwartz distributions [8]. Then, by the theorem on local structure of the distributions (see $\left[9\right.$, chap.1, n.2]) there exist a function $g(x) \in L_{\infty}(e)$ and an integer $k \geq 0$ such that

$$
f(x)=D_{1}^{k} \ldots D_{n}^{k} g(x),
$$

where $x \in e$, the set $e$ is compact in $G \subset R^{n}$.

Let $v(x) \in D(e)$, from $(26)$ and $(21)$ one has

$$
\iint_{e} \sum_{i=1}^{2} \frac{\partial}{\partial x_{i}}\left(k_{i}(x) \frac{\partial u}{\partial x_{i}}\right) v(x) d x=-\iint_{e} g(x) \widetilde{v}(x) d x
$$

where

$$
\widetilde{v}(x)=D_{1}^{k} D_{2}^{k} v(x) \quad(n=2) .
$$

We have $\widetilde{v}(x) \in D(e), g(x) \in L_{2}(e)$. Therefore, the equation (27) has the form (21), (22). Then one may repeat the procedure used above and obtains the following

Theorem 3. Let the coefficients $k_{i}(x)$ of the problem (19) belong to the space $W_{\infty}^{m-1}(G)$, satisfying the condition (20), $m=2,3$, and let the right-hand side $f(x) \in W_{2}^{(-l)}(G)$. Then the solution $y$ of the scheme (23) or (24) converges to the GS (22) u of the problem (19) in the grid norm $W_{2}^{1}(\omega)$ with the rate $O\left(h^{m / 2}\right)$, that is, there is a number $M$ such that

$$
\|y-u\|_{1, \omega} \leq M|h|^{m / 2}\|u\|_{m, G},
$$

where the constant $M$ is independent of $h$ and $u(x)$.

\section{REFERENCES}

[1] Barrett J. and Knabner P., Finite element approximation of the transport of reactive solutes in porous media, SIAM J. Numer. Anal. 34 (1) (1997) 201-227.

[2] Dang Quang A, Approximate method for solving an elliptic problem with discontinuous coefficients, J. of Comp. and Applied Math. 51 (1994) 193-203.

[3] Dörfler W., Uniform a priori estimates for singularly perturbed elliptic equations in multidimensions, SIAM J. Numer. Anal. 36 (5) (1999) 1878-1900.

[4] Hoang Dinh Dung, Difference schemes for generalized solutions of some elliptic differential equations, I, J. of Comp. Science and Cybern. 15 (1) (1999) 49-61.

[5] Marchuk G. I., Mathematical Modeling in the Environment Problems (Russian), Science, Moscow, 1982.

[6] Pani A. K., An $H^{1}$-Galerkin mixed finite element method for parabolic partial differential equations, SIAM J. Numer. Anal. 35 (2) (1998) 712-727.

[7] Samarski A. A., Lazarov R. D., Makarov V.L., Difference Schemes for Generalized Solutions of Differential Equations (Russian), "Univ.", Moscow, 1987.

[8] Schwartz L., Théorie des Distributions, Hermann, Paris, 1978.

[9] Vladimirov V.S., Generalized Functions in Mathematical Physic, Mir., Moscow, 1979.

Received May 18, 1999

Revised April 26, 2000

Institute of Mathematics, Hanoi, Vietnam. 